\title{
ARTICLE OPEN CRISPR/Cas9-based genetic correction for recessive dystrophic epidermolysis bullosa
}

Beau R Webber ${ }^{1,5}$, Mark J Osborn ${ }^{1,2,3,4,5}$, Amber N McElroy ${ }^{1}$, Kirk Twaroski ${ }^{1}$, Cara-lin Lonetree ${ }^{1}$, Anthony P DeFeo ${ }^{1}$, Lily Xia ${ }^{1}$, Cindy Eide ${ }^{1}$, Christopher J Lees ${ }^{1}$, Ron T McElmurry ${ }^{1}$, Megan J Riddle ${ }^{1}$, Chong Jai Kim ${ }^{4}$, Dharmeshkumar D Patel ${ }^{1}$, Bruce R Blazar ${ }^{1,2}$ and Jakub Tolar ${ }^{1,2,4}$

Recessive dystrophic epidermolysis bullosa (RDEB) is a severe disorder caused by mutations to the COL7A1 gene that deactivate production of a structural protein essential for skin integrity. Haematopoietic cell transplantation can ameliorate some of the symptoms; however, significant side effects from the allogeneic transplant procedure can occur and unresponsive areas of blistering persist. Therefore, we employed genome editing in patient-derived cells to create an autologous platform for multilineage engineering of therapeutic cell types. The clustered regularly interspaced palindromic repeats (CRISPR)/Cas9 system facilitated correction of an RDEB-causing COL7A1 mutation in primary fibroblasts that were then used to derive induced pluripotent stem cells (iPSCs). The resulting iPSCs were subsequently re-differentiated into keratinocytes, mesenchymal stem cells (MSCs) and haematopoietic progenitor cells using defined differentiation strategies. Gene-corrected keratinocytes exhibited characteristic epithelial morphology and expressed keratinocyte-specific genes and transcription factors. iPSC-derived MSCs exhibited a spindle morphology and expression of CD73, CD90 and CD105 with the ability to undergo adipogenic, chondrogenic and osteogenic differentiation in vitro in a manner indistinguishable from bone marrow-derived MSCs. Finally, we used a vascular induction strategy to generate potent definitive haematopoietic progenitors capable of multilineage differentiation in methylcellulose-based assays. In totality, we have shown that CRISPR/Cas9 is an adaptable gene-editing strategy that can be coupled with iPSC technology to produce multiple gene-corrected autologous cell types with therapeutic potential for RDEB.

npj Regenerative Medicine (2016) 1, 16014; doi:10.1038/npjregenmed.2016.14; published online 8 December 2016

\section{INTRODUCTION}

Recessive dystrophic epidermolysis bullosa (RDEB) is a monogenic disorder resulting from mutations in the type VII collagen gene (COL7A1) on chromosome 3 . The mutational profile can be heterogeneic in regards to position and can encompass homozygous or compound heterozygous alterations. ${ }^{1}$ The resultant loss of the functional type VII collagen protein (C7) at the dermalepidermal junction compromises the integrity of the attachment of the epidermis to the dermis, resulting in severe blistering, fibrosis and a predisposition to squamous cell carcinoma. Non-cutaneous manifestations, including corneal and oesophageal lesions, further contribute to a pathogenic state leading to a multi-decade decrease in life expectancy.

Treatment for RDEB includes palliative bandaging of active wounds and pain management, as well as allogeneic and autologous cellular therapy. Palliation is non-curative, and cellular therapy can include localised injection of type VII collagenexpressing cells and/or systemic infusion of haematopoietic stem/progenitor cells (HSPCs) that repopulate the host with donor-derived cells. ${ }^{2}$ Keratinocytes and fibroblasts represent the major C7 producing cells of the skin; however, their poor in vitro proliferative and expansion properties as primary cells limit their therapeutic potential and impact. Mesenchymal stromal/stem cells (MSCs) have been used as a supportive therapy and possess wound migratory potential and the ability to actively participate in, as well as to orchestrate, healing. ${ }^{3,4}$ Similar to other primary cells, primary bone marrow-derived MSCs can senesce and lose their beneficial properties with in vitro expansion.

Towards mediating systemic effects, allogeneic haematopoietic cell transplant (HCT) has been employed. HCT has resulted in significant, but neither uniform nor complete, outcomes. ${ }^{5}$ For each modality, the use of allogeneic cells limits efficacy. Locally injected cells appear to persist transiently, likely due to immune clearance, necessitating repeated injections that is limiting in terms of the difficulty in long-term culture/maintenance, surface area able to be treated, and availability of allogeneic cells that can be obtained, archived and expanded for subsequent injections. ${ }^{6}$ $\mathrm{HCT}$ can result in graft-versus-host disease that can cause severe side effects, making the use of autologous cells highly desirous. To realise the potential of such an approach, we set out to determine whether an RDEB patient's COL7A1 gene defect could be restored to wild-type status in a population of cells that could be utilised as a template for sustainable multilineage progeny generation.

Two major platforms exist for facilitating gene correction: gene therapy and gene editing. Gene therapy for RDEB has centred primarily on lentiviral gene transfer of a copy of the COL7A1 CDNA, expression of which is governed by exogenous regulatory elements. ${ }^{7,8}$ While this strategy meets the need for autologous

\footnotetext{
'Department of Pediatrics, Division of Blood and Marrow Transplantation, University of Minnesota, Minneapolis, MN, USA; ${ }^{2}$ Stem Cell Institute, University of Minnesota, Minneapolis, MN, USA; ${ }^{3}$ Center for Genome Engineering, University of Minnesota, Minneapolis, MN, USA and ${ }^{4}$ Asan-Minnesota Institute for Innovating Transplantation, Seoul, Republic of Korea.

Correspondence: J Tolar (tolar003@umn.edu)

${ }^{5}$ These authors contributed equally to this work.

Received 1 August 2016; revised 17 October 2016; accepted 26 October 2016
} 


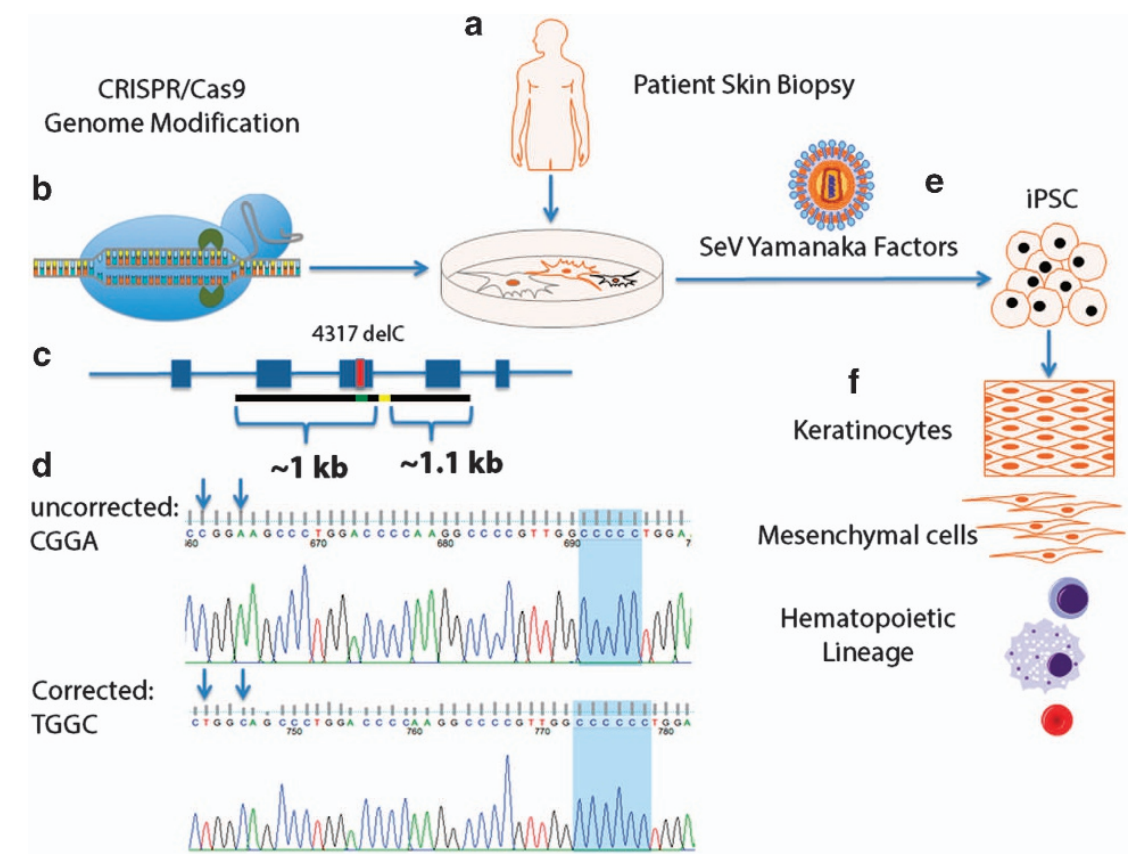

Figure 1. Experimental schema for gene correction and cellular engineering. (a) A punch biopsy was obtained for primary fibroblast cell derivation. (b) The CRISPR/Cas9 gene-editing platform was employed for $4317 \mathrm{delC}$ COL7A1 gene correction. (c) COL7A1 locus and gene repair template. The 4317delC mutation is indicated with a red box. The donor template was a plasmid containing $\sim 1 \mathrm{~kb}$ of homology to the target sequence and flanked a floxed PGK puromycin selection cassette (yellow box). The cytosine to restore proper genotype and two silent polymorphisms were introduced into the donor arm and is indicated with a green box. (d) COL7A1 locus correction. Sanger sequence of uncorrected cells before treatment showing a deletion of a single cytosine and unmodified base sequences (top). Subsequent CRISPR/Cas9 mediated repair by the donor resulted in restoration of the deleted cytosine (shaded in blue) and incorporation of engineered marker SNPs (blue arrows). (e) The corrected fibroblasts were reprogrammed to pluripotency using Sendai virus delivery of the reprogramming factors. (f) iPSCs served as a template for directed differentiation into keratinocytes, mesenchymal stem cells and haematopoietic progenitors.

cellular engineering, there are significant hurdles to this approach. The large size of the cDNA can negatively impact viral titres making manufacturing, production and efficient gene delivery rates suboptimal. Further, the integrating properties of vectors capable of long-term gene expression represent a risk for insertional mutagenic-derived adverse events. This fact is particularly relevant given that RDEB patients are at an increased risk for squamous cell carcinoma, thus potentially placing cutaneous cells in a pre-malignant state that are less tolerant to the genomic perturbation that accompanies integrating vectors. ${ }^{9}$ In addition, the artificial expression cassette components are not subject to the normal cellular gene regulatory environment. This point is highly relevant, as perturbation of ECM protein expression has been shown to impact the cellular microenvironment, and the long-term effect of supra-physiological COL7A1 expression is unknown. These considerations make gene editing the preferred methodology for autologous cell precision correction in situ that mitigates genomic toxicity and maintains the endogenous cellular gene expression command and control system.

Gene targeting at translational efficiency requires site-specific reagents that cleave the DNA helix, and there are multiple candidates capable of accomplishing this. Zinc finger nucleases and transcription activator-like effector nucleases function as dimeric proteins that co-localise at a target site and mediate a double-stranded DNA break. ${ }^{10-12}$ Meganucleases are a monomeric protein specified for a unique sequence utilising a bacterial endonuclease as the engineering template. ${ }^{13}$ The clustered regularly interspaced palindromic repeats (CRISPR)/Cas9 reagent is a two-component system consisting of the Cas9 nuclease that conjugates with a small RNA transcript termed a guide RNA (gRNA). This complex interacts with a target sequence consisting of 15-20 nucleotides. Once a DNA break is generated, genome modification by homology-directed repair (HDR) can occur using an exogenous donor DNA species as the repair template.

Here we utilised the CRISPR/Cas9 system to facilitate gene repair in cells from an RDEB patient. Correction of COL7A1 fibroblasts was achieved, and these fibroblasts were used as a renewable template for patient-specific induced pluripotent stem cell (iPSC) derivation. To capitalise on the ease of continuous propagation and broad differentiation potential of iPSCs, they were employed in a therapeutic engineering strategy to generate keratinocytes, MSCs and haematopoietic cells. This multilineage approach represents a strategy for broad therapeutic use in support of combinatorial systemic and localised interventions. ${ }^{14}$

\section{RESULTS}

The overall experimental schema is detailed in Figure 1 and is a strategy that employed patient-derived fibroblasts that were precisely modified with a CRISPR/Cas9 mutation-specific reagent. Cells were induced to pluripotency with Sendai viral, footprint-free reprogramming and differentiated into effector lineages.

\section{CRISPR/Cas9 gene correction}

We generated three candidate gRNAs proximal to the 4317 delC COL7A1 gene mutation characterised by a single cytosine deletion (Supplementary Figures S1a and b) for testing with the S. pyogenes Cas9 that was delivered as DNA expression cassettes (Supplementary Figures S1c and d). Using the Surveyor assay, ${ }^{15}$ all three candidates resulted in cleavage products consistent with Cas9 activity and DNA repair by non-homologous end joining (Supplementary Figures S1e and f). Because fibroblasts are a major contributor of C7 in the dermis, we deployed CRISPR/Cas9 reagents for gene correction using a targeting strategy comprised 
Table 1. Off-target activity analysis

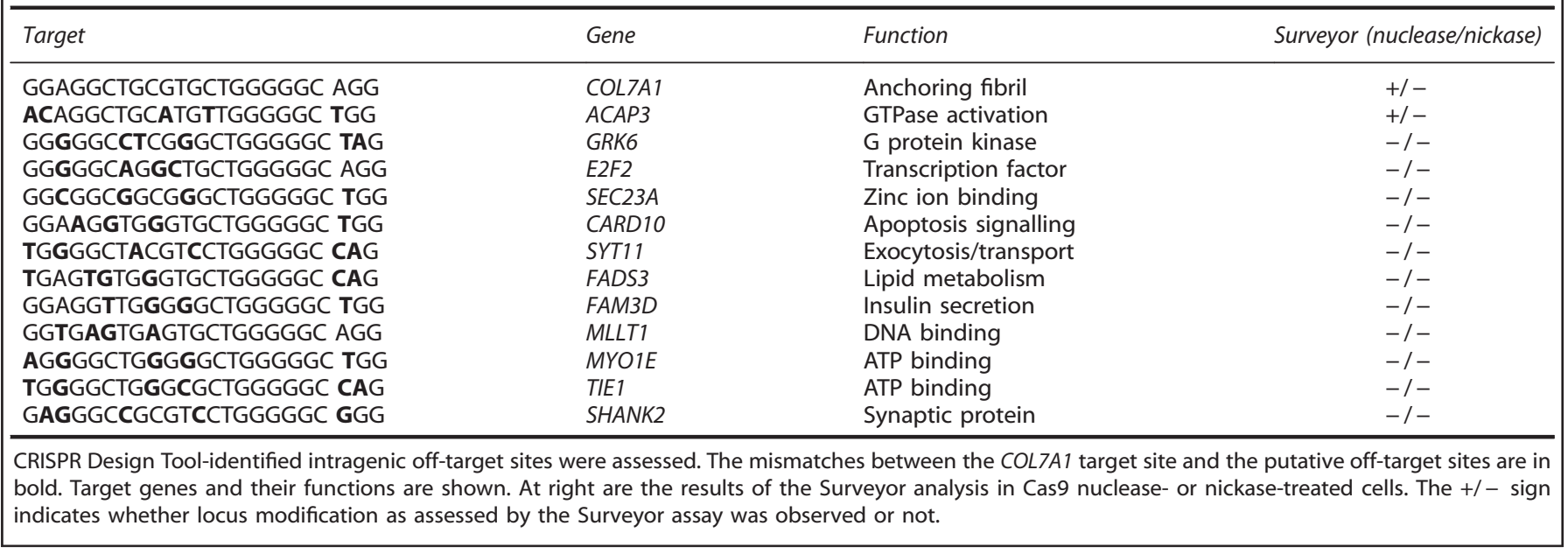

of three plasmids: Cas9 nuclease or nickase, the C7 gRNA, and an exogenous donor repair template (Figure 1c and Supplementary Figure S2a). The Cas9 nuclease generates double-stranded DNA breaks while the nickase version, due to inactivation of the Cas9 $\mathrm{HNH}$ domain, cleaves a single strand of DNA. ${ }^{16} \mathrm{~A}$ double-stranded DNA donor template was constructed consisting of homology arms of $\sim 1 \mathrm{~kb}$ flanking a floxed puromycin drug resistance gene in such a way that it would be inserted into an adjacent intron for selection and subsequent cre-recombinase-mediated removal (Figure 1c and Supplementary Figure S2a). To allow for unambiguous determination of donor-derived HDR, we included silent polymorphisms within the right donor arm (Supplementary Figure S2a). The donor fragment and the nuclease or nickase version of Cas9 and gRNA 2 were electroporated into fibroblasts that were then puromycin-selected in bulk. The puromycinresistant cells were screened for HDR using an 'inside-out' PCR strategy, with a primer inside the donor and outside of the right homology arm.

Bulk population cells were plated at low density to allow for clonal selection, expansion and screening, and 17 total clones were obtained. Four clones that received the Cas9 nickase, and eight treated with Cas9 nuclease, showed HDR at the genomic level (Figure 1d and Supplementary Figure S2b). The puromycin cassette was subsequently removed using cre-mRNA, which resulted in a small loxp footprint in the intron (Supplementary Figure S2c). Screening at the cDNA level showed gene correction and donor polymorphism presence in COL7A1 gene transcripts (Supplementary Figure S2d). These data show the ability of the CRISPR/Cas9 to mediate gene repair in primary fibroblasts obtained from an RDEB patient that, importantly, retained a normal morphology and karyotype (Supplementary Figures $\mathrm{S} 3 \mathrm{a}$ and $\mathrm{b}$ ).

An important consideration for the employment of programmable gene-editing reagents is their specificity for the intended gene target. Because of the presence of sequences of partial homology to the bona fide target, we utilised a predictive in silico modelling algorithm to identify potential off-target sites (Table 1). To determine whether promiscuous CRISPR/Cas9 cutting occurred, we screened the putative off-target sites using the Surveyor assay. We observed one off-target site in the ACAP3 gene (Supplementary Figure S4) that functions as a GTPase activating protein. ${ }^{17}$ Importantly, the nickase version of Cas9 that preferentially promotes HDR ${ }^{15}$ did not show any mutagenic non-homologous end joining at this locus (Supplementary Figure S4) and we therefore employed nickase-corrected clones for reprogramming to pluripotency.

\section{RDEB gene-corrected iPSCs}

Utilising Sendai virus-based reprogramming, we obtained transgene-free, gene-corrected, karytotypically normal iPSCs (Supplementary Figures S3c and d). Resultant iPSC clones were positive for pluripotent markers at the protein (Supplementary Figure S5) and gene expression levels (Supplementary Figure S6a). As further verification of successful reprogramming, the OCT4 and NANOG gene promoters were observed to be hypomethylated, and in vivo teratomas derived from gene-corrected iPSCs contained representative tissues from all three germ layers. (Supplementary Figures S6b and c).

These results demonstrate that CRISPR/Cas9 genome modification and Sendai virus reprogramming allow for precision repair and iPSC generation. We subsequently employed this population of cells to derive therapeutic cell types suitable for cellular therapies for RDEB.

\section{Production of keratinocytes from gene-corrected iPSCs}

Treatment of the chronic wounds experienced by RDEB patients could be bolstered by the localised delivery of gene-corrected keratinocytes. To demonstrate that iPSCs derived from CRISPR/ Cas9 gene-corrected RDEB fibroblasts are capable of differentiating into therapeutically relevant cell populations in vitro, we utilised protocols to produce keratinocytes under fully defined, feeder-free conditions. ${ }^{18-20}$ Two-dimensional culture of iPSCs in the presence of retinoic acid (RA) and bone morphogenic protein4 (BMP-4) resulted in the formation of cells with characteristic epidermal morphology (Figure 2). Compared with undifferentiated iPSCs, differentiation cultures enriched for keratin-expressing epidermal cells by rapid attachment to type IV collagen-coated tissue culture plates exhibited significantly elevated expression of mRNAs associated with commitment to the keratinocyte lineage as well as elevated expression of Col7A1 mRNA (Figure 2b). Concordant with keratinocyte morphology and mRNA expression profile, iPSC-derived keratinocyte cultures contained a high frequency of cells positive for keratin-5 (Krt5), keratin-14 (Krt14) and transcription factor p63 (Figure 2c). These data show the ability of CRISPR/Cas9 gene-corrected iPSCs to successfully differentiate into keratinocytes in vitro under fully defined, xenofree culture conditions amenable for clinical translation.

\section{Production of MSCs from gene-corrected iPSCs}

MSCs are mesoderm-derived, fibroblastic cells present within many tissues, including bone marrow and adipose. There is substantial evidence that MSCs or MSC-derived cells can enhance 
a

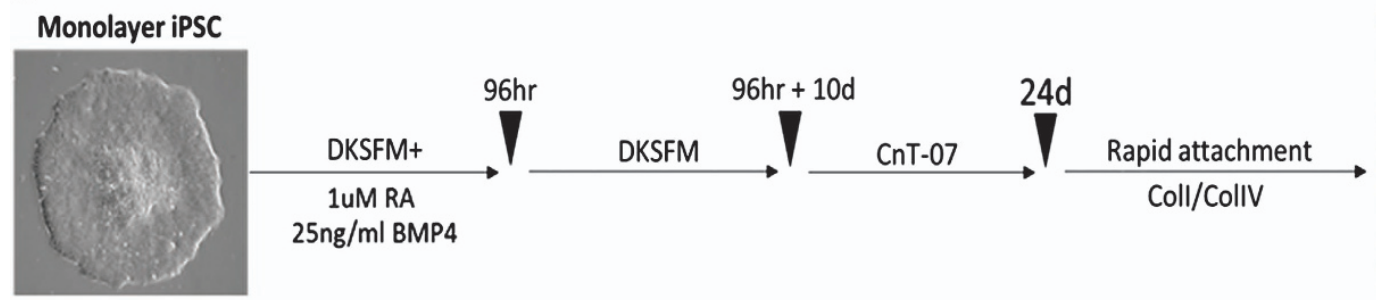

b

Krt5

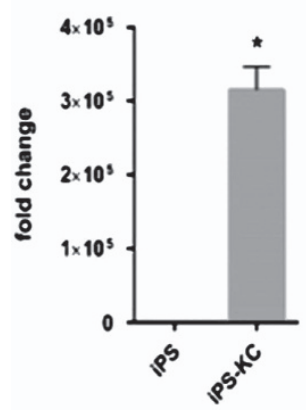

Col17A1

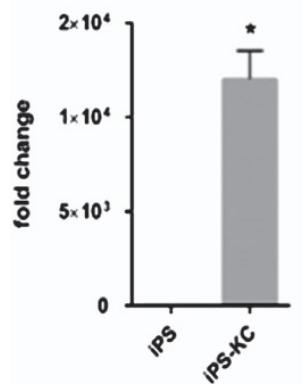

Krt15

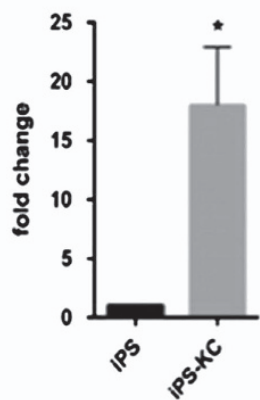

Col7A1

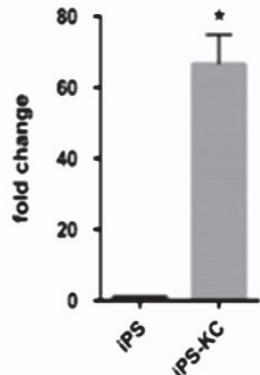

C
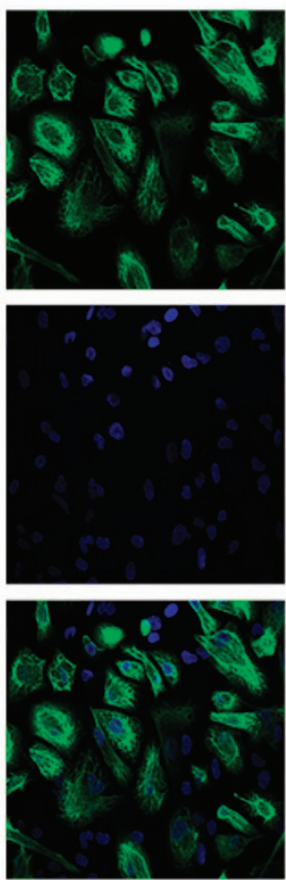

KRT14
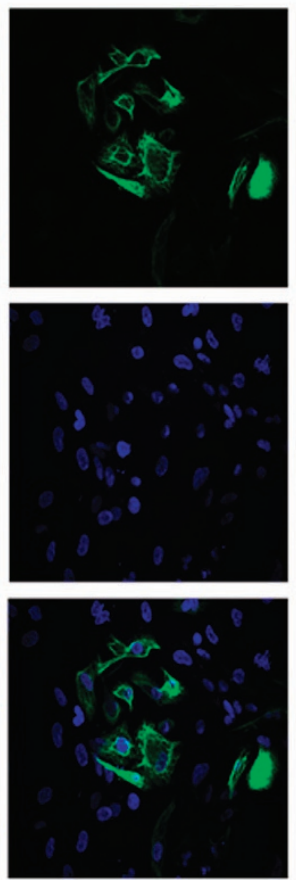

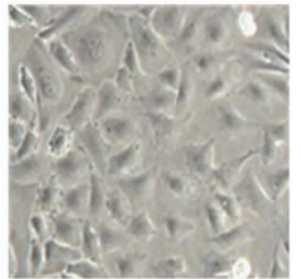

p63
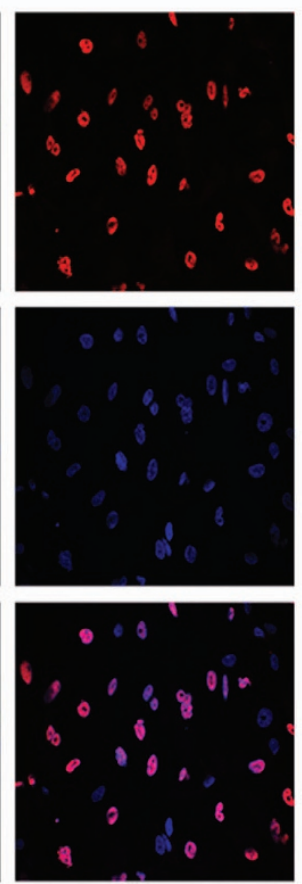

Figure 2. Keratinocyte generation. (a) Derivation schema. iPSC colonies in a six-well plate were exposed to RA and BMP-4 in defined keratinocyte serum-free media with a transition to the CnT-07-defined keratinocyte media. Keratinocytes were enriched by exploiting their ability to rapidly attach to collagens I- and IV-coated plates. (b) Keratinocyte gene expression analysis. TaqMan RT-qPCR was performed for keratin 5 and 15, COL17A1 and COL7A1 (three different experiments) and compared with parental iPSCs. (c) Keratinocyte immunofluorescence. Gene-corrected, iPSC-derived cells were stained with anti-KRT5, KRT15 or p63 antibodies (top). Middle panel is DAPI nuclear staining and bottom is merged images that are representative of 3-4 independent experimental replicates. BMP, bone morphogenic protein; RA, retinoic acid; RT-qPCR, quantitative PCR with reverse transcription.

wound healing via modulation of wound microenvironment, immunomodulation, or by direct integration into cutaneous tissues after transplant. ${ }^{21-26}$ At present, targeted gene editing in primary human MSCs has not been demonstrated, leading us to pursue directed differentiation of COL7A1 gene-corrected iPSCs to MSCs in vitro. ${ }^{27}$ COL7A1 gene-corrected iPSCs were exposed to MSC media supplemented with platelet-derived growth factor (PDGF)-AB, basic fibroblast growth factor (bFGF), and epidermal growth factor (EGF) until iPSC colony morphology was lost and outgrowth of cells with fibroblastic morphology was observed (Figure 3a). At this stage the cells were passaged from the matrigel substrate to gelatin-coated plastic tissue culture vessels. We observed that cell survival and re-plating during passages 1-3 is substantially enhanced in the presence of Rho-associated protein kinase (ROCK) inhibitor, allowing for more consistent establishment of robust iPSC-MSC cultures capable of expansion with minimal senescence (Supplementary Figure S7a). Between passages 3-5, uniform iPSC-MSC cultures were obtained and flow cytometric analysis demonstrated the expression of MSC markers CD73, CD90 and CD105 (Figure 3b). Furthermore, we show that COL7A1-corrected iPSC-derived MSCs were able to undergo robust tri-lineage differentiation to adipocytes, chondrocytes and osteoblasts in vitro (Figures $3 \mathrm{c}-\mathrm{e}$ ); a defining characteristic of primary MSCs. ${ }^{28}$ Collectively, these data show that COL7A1 gene-corrected iPSCs are capable of producing MSCs in vitro, and that these iPSC-MSCs are indistinguishable from primary human MSCs in their morphology, cell surface antigen profile, and their capacity for tri-lineage differentiation and retain the ability to self-renew in culture (Figure 3 and Supplementary Figure S7). Moreover, the gene-corrected iPSC-derived MSCs but not parental uncorrected fibroblasts showed collagen type VII protein expression (Supplementary Figure S7c).

Production of definitive haematopoietic progenitors from genecorrected iPSCs

Although cutaneous blistering is the most apparent pathology observed in patients with RDEB, it is the systemic manifestationsincluding mucosal blistering-that are often the most destructive and life-threatening and which cannot be resolved by localised grafting of epidermal cell types such as keratinocytes or fibroblasts. Our group and others have previously reported the 
a

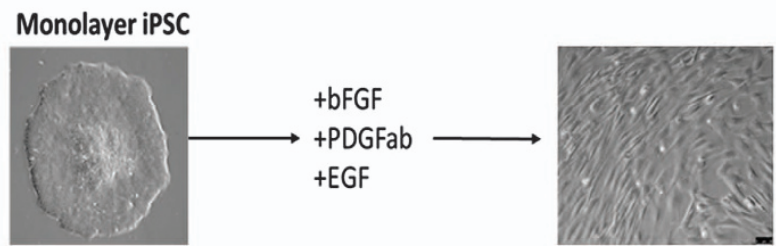

b
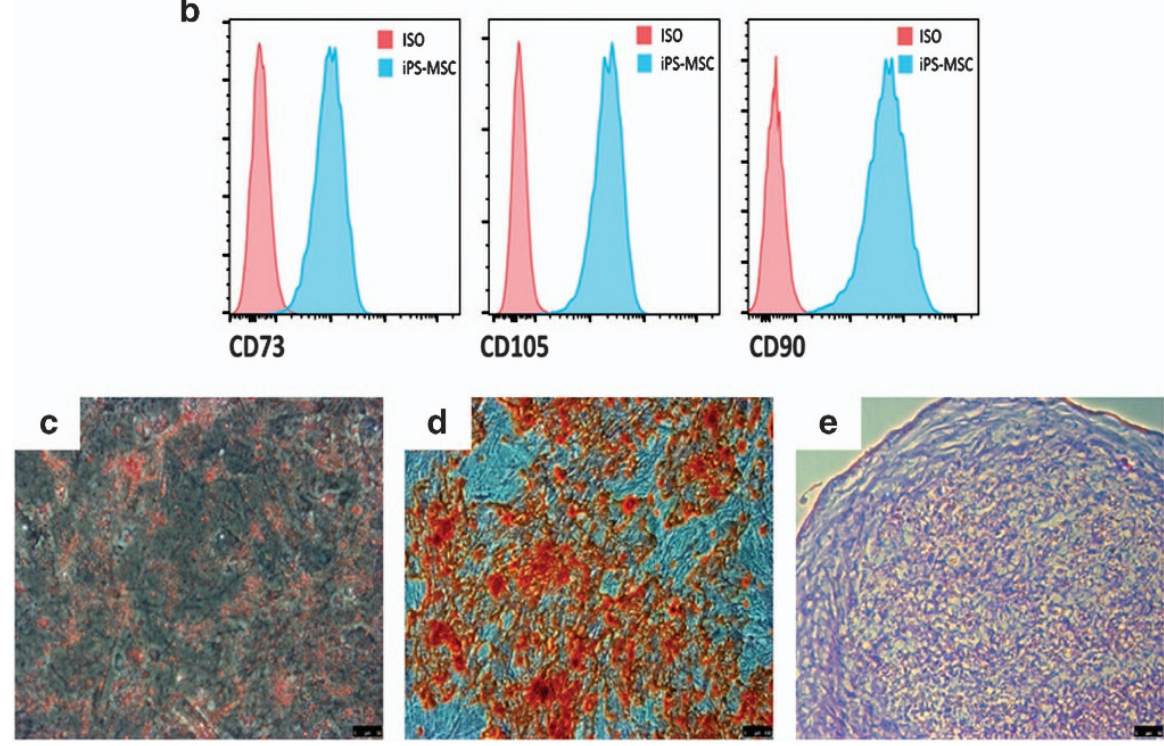

Figure 3. Mesenchymal stem cell derivation. (a) MSC differentiation. Mono-layer iPSCs were subjected to bFGF, PDGFab and EGF resulting in differentiation to a cell population with spindle-shaped morphology (right). (b) FACS analysis. Passage 3 MSCs were analysed for cell surface expression of CD73, CD105, and CD90 ( $n=3$ experiments), and histogram analysis is shown in blue. Isotype antibody control FACS histograms are shown in pink. (c,d) Tri-lineage differentiation. (c) Oil red-O staining demonstrating the ability of iPSC-derived MSCs to form adipose cells. (d) Alizarin red staining of osteogenic progeny. (e) Toluidine blue staining of chondrogenic cells from MSCs. (c-e) Representative images of at least two different MSC pools and $n=3-4$ replicates. FACS, fluorescence-activated cell sorting.

amelioration of systemic RDEB pathology by HCT that is associated with a substantial risk of morbidity and mortality during the preparative and post-transplant period. ${ }^{29,30}$ Therefore, the generation of gene-corrected HSPCs would be a highly desirable approach for autologous therapy. Although targeted gene-editing in severe combined immune deficiency-repopulating human HSPCs has been demonstrated, the efficiency is not yet robust enough for models such as RDEB that do not confer a selective or proliferative advantage to the modified cell(s). ${ }^{31-33}$ Therefore, we undertook efforts to advance the understanding of in vitro HSPC generation using a translational workflow under serum and feeder-free conditions. Protocols for serum-free haematopoietic differentiation are well described, yet in each of these reports the starting iPSCs are cultured on xenogeneic murine feeder cells, which is suboptimal for clinical translation. ${ }^{34-36}$ Our efforts to implement these protocols to feeder-free iPSC cultures were met with limited success, and we observed that embryoid body formation, growth and production of CD34+ hemogenic progenitors was poor (Supplementary Figure S8). In light of these observations we sought to optimise the differentiation protocol to allow robust generation of haematopoietic progenitors under fully defined, serum-free, xeno-free conditions. We employed the nutrient-rich, human serum albumin-based media TeSR1 for maintenance of feeder-free cells, and generated embryoid bodies $(E B)$ in the presence of the haematopoietic progenitor medium StemPro34. Haematopoietic induction frequency is highest when cells transit through an EB stage, and we observed that the abrupt transition from TeSR1 to StemPro34 resulted in poor haematopoietic cell derivation (Supplementary Figure S8). Therefore, we pursued EB formation in StemPro34 supplemented with dilutions of TeSR 1 and found that EB formation was enhanced substantially (Supplementary Figure S8). In support of our ultimate goal of feeder-free iPSCs with differentiation carried out in fully defined media, we sought to identify the conditions for elimination of TeSR1 media that contains undefined human serum albumin. We therefore employed animal-free polyvinyl alcohol essential lipid (APEL) as a base media that allowed for sustained growth of EB after TeSR1 removal and robust production of CD34+ haematopoietic progenitors at day 8 (Figure $4 a) .{ }^{37}$ As part of this procedure we modulated the commitment phase of blood development by combining Activin/Nodal inhibition and GS3K $\beta$ inhibition at the mesoderm phase of differentiation in order to bias the system towards definitive haematopoiesis. ${ }^{38}$ A substantial portion of the CD34+ fraction at day 8 was negative for the mesenchymal marker CD73 and the vascular marker CXCR4, representing a robust population of hemogenic precursors (Figure $4 \mathrm{~b}) .^{34}$ We further confirmed the definitive potential of EB-derived CD34+ cells via demonstration of T-lineage specification upon co-culture with OP9-DLL4 (Supplementary Figure S9). ${ }^{34-36,39}$ However, when assessed directly in vitro by methylcellulose-based haematopoietic colony-forming unit (CFU) assay, the day 8 EB CD34+ fraction exhibited a markedly limited myeloid potential, indicating that further maturation was required to complete ultimate specification to committed HSPCs (Figure 4c). Given the complex niche in which definitive HSPCs arise during development, we next sought to recapitulate that environment in vitro. To accomplish this we employed a supportive human vascular stromal endothelial cell population that expresses the adenovirus E4ORF1 gene and has 

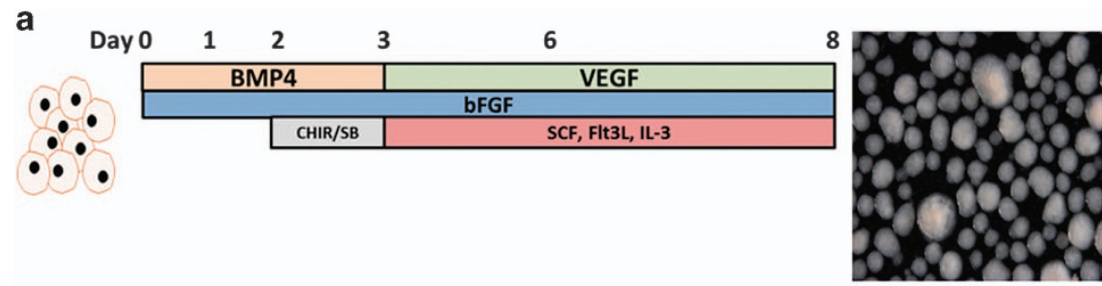

b

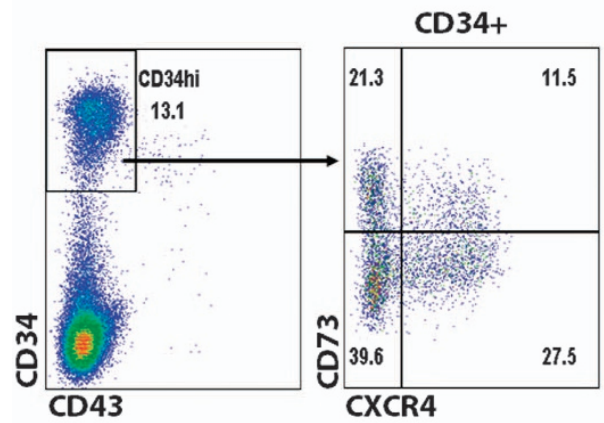

C

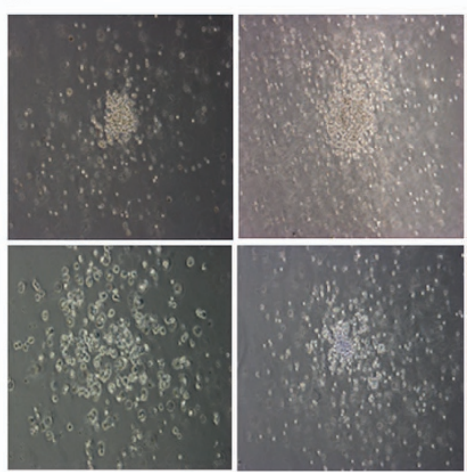

Figure 4. Haematopoietic differentiation. (a) Culture condition schema. Embryoid bodies were formed from iPSCs in the presence BMP4, VEGF, bFGF, CHIR99021 and SB431542, and haematopoietic cytokines (Flt3-ligand, SCF and IL-3) over the course of 8 days. A representative image of EBs are shown at right. (b) FACS phenotype of EB-derived CD34. Dissociated EBs were analysed by FACS for CD73 and CXCR4 in the CD34 high-expressing population. (c) Methylcellulose CFU assay. CD34 cells were embedded in methylcellulose, and an image of the dominating CFU-G/M colony types are shown. The analyses/experiments are from at least four different differentiation procedures. FACS, fluorescence-activated cell sorting.

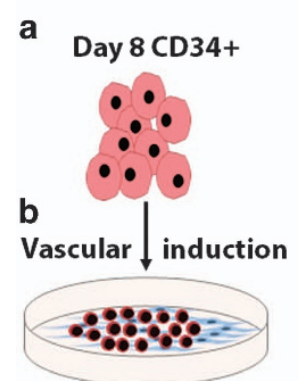

Day 8+7 MPP
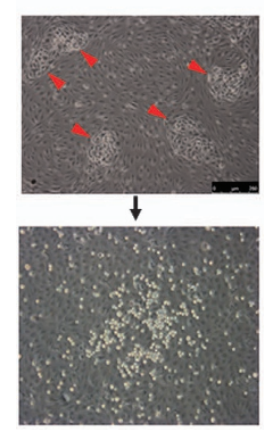

C VeraVec only

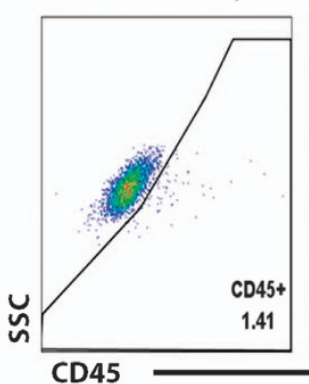

d

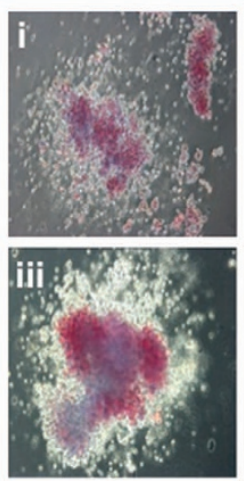

D8+7 co-culture
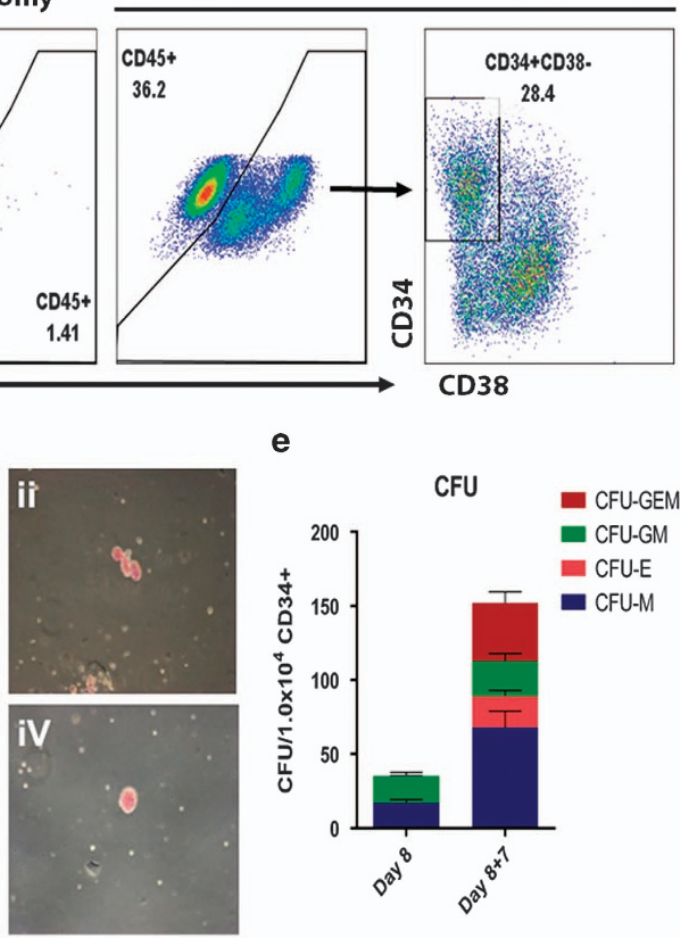

e

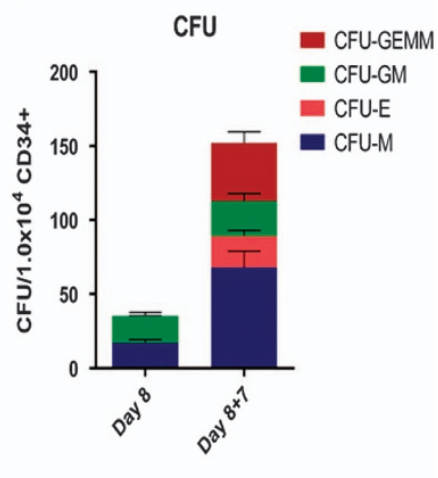

Figure 5. Haematopoietic differentiation and vascular induction. (a) Experimental design. EBs formed over 8 days (as shown in this figure), were dissociated, sorted for CD34 cells, and then incubated on the VeraVec endothelial cell line expressing the E4ORF1 gene. (b) Multipotent haematopoietic progenitors. Upon vascular induction, small clusters of budding hemogenic cells (red triangles) were evident by light microscopy and gave rise to non-adherent haematopoietic progenitors. (c) FACS phenotype of iPSC CD34s that underwent vascular induction. Day 8 CD34 from EBs cultured on VeraVec cells for 1 week was analysed by flow cytometry for CD34, 48 and 45 . (d) Vascular induction CFU. CD34 CD45+ cells isolated from the VeraVec feeder cells were cultured in methylcellulose, and representative colonies for CFU-GEMM (i and iii) and CFU-E (ii and iv) are shown. (e) CFU quantification. Three independent experiments were analysed and quantified for the number of lineage-specific colonies formed for CD34 cells derived +/- vascular induction. CFU-GEMM, colony-forming unit-granulocyte, erythrocyte, monocyte/macrophage, megakaryocyte; CFU-GM, colony-forming unit granulocyte- monocyte/macrophage; CFU-E, colony-forming unit erythroid; CFU-M, colony-forming unit monocyte/macrophage; FACS, fluorescence-activated cell sorting. 
been shown to support endothelial to HSPC derivation in vitro. ${ }^{40,41}$ We generated EB-derived mature CD34+ cells as before (Figure 5a), and at day 8 purified CD34+ hemogenic precursors were co-cultured with the vascular stroma and subsequently underwent endothelial to haematopoietic transition to form rounded haematopoietic cells that contained a subpopulation of CD45+CD34+CD38- haematopoietic progenitors (Figures 5b and c). In CFU assays, day 8 EB-derived CD34+ cells that underwent vascular induction showed a broader colony differentiation potential (Figure $5 \mathrm{~d}$ ). In aggregate, day 8 EBs with 7-day co-culture on a supportive vascular endothelial matrix resulted in both a significant increase in colony frequency and an expansion in multilineage potential compared with day 8 EBs alone (Figure 5e). These data demonstrate that COL7A1-corrected iPSCs represent a platform for the in vitro production of definitive haematopoietic progenitors capable of producing multiple blood lineage cell types in CFUs that represent an advance towards true HSPC in vitro generation.

\section{DISCUSSION}

RDEB is associated with a loss of the functional integrity of the dermal-epidermal junction, which results in painful erosions and blistering. The disease process is not limited to the cutaneous manifestations; therefore, a platform for combinatorial regenerative medicine must possess attributes of tissue plasticity, migratory properties and an effector lineage capable of C7 deposition. To date, infusions of allogeneic HSCs and progenitor cells, fibroblasts, keratinocytes and MSCs have all been pursued clinically. Although HCT confers a lifelong supply of donorderived cells, the mechanism for wound repair remains poorly understood. Further, areas of the skin are often refractory to benefit from HCT, and supportive localised therapy is required for more penetrant effects. Fibroblasts and keratinocytes are the primary C7-producing cells in the skin and have been utilised for localised, supportive therapy as have MSCs, which possess the capacity to home to wounded tissue and deposit $C 7 .{ }^{42}$ No one cell type is endowed with each of these beneficial properties, and post-injection cellular persistence is transient in nature requiring repetitive delivery necessitating a manufacturing process reliant on long-term culture and expansion that may drive the cells towards senescence. Autologous RDEB iPSCs that are derived from patient cells represent a potential solution to this shortcoming provided that a corrected COL7A1 gene sequence is present. ${ }^{18,43}$ To maximise the potential of this approach, we coupled cellular reprogramming with precision gene correction, representing a platform for production of autologous cell types for regenerative medicine. ${ }^{44-47}$

Members of our group, ${ }^{44}$ and Chamorro et al. ${ }^{47}$ have utilised transcription activator-like effector nucleases for COL7A1 gene correction in fibroblasts and keratinocytes, respectively. In a study by Izmiryan et al. meganucleases with an integrase-deficient lentiviral vector were used to mediate gene repair in keratinocytes and fibroblasts. ${ }^{45}$ Sebastiano et $a .^{46}$ and Wenzel et al. ${ }^{48}$ used iPSCs as a template for gene correction without classically engineered nucleases. They used a flip recombinase in murine cells or a pro-recombinogenic corrective donor construct alone delivered on an adeno-associated viral vector in human cells, respectively. ${ }^{46}$ These studies show that the RDEB genotype and phenotype are amenable to correction by genome engineering. Building off these studies we set out to resolve two gaps in the current RDEB cellular and genome-engineering procedures: (i) integration-free derivation and subsequent feeder-free maintenance of iPSCs; and (ii) the utilisation of genetically corrected iPSCs for generation of multiple therapeutic cell types under defined conditions as proof of concept for multilineage cellular therapy (Figure 1). Towards the former, we and others have used integrating vector delivery of the iPSC reprogramming factors that either remain in the genome or require subsequent removal. ${ }^{43,44}$ In the latter, our previous work relied on in vivo teratoma formation that occurs in poorly defined conditions that are not able to be controlled ex vivo. ${ }^{44}$ Sebastiano et al. report efficient generation of minimally heterogeneic keratinocytes; however, this cell population does not represent a durable modality for broad use. As such, in the current work we describe a gene correction strategy employing the CRISPR/Cas9 system that resulted in gene correction in fibroblasts that are of immediate value for localised C7 production. We then employed the Sendai reprogramming methodology to generate iPSCs that were used for subsequent derivation of multilineage cell types with therapeutic value.

Three CRISPR/Cas9 targeting candidates were tested, with one employed for gene correction in comparative studies with the nuclease or nickase version of the $S$. pyogenes Cas9 (Supplementary Figure S1). Similar to previous observations, we observed a lower overall rate of HR using the nickase (4/12 clones showing HDR versus $8 / 12$ for the nuclease); however, the preferential repair of DNA nicks by the HR pathway adds a further layer of specificity to the engineering process. This is highlighted by the observation of an off-target event at the ACAP3 gene with the nuclease, but not nickase, version of Cas9 (Table 1 and Supplementary Figure S4). The derivation of clones was facilitated by our donor design strategy that included a puromycin cassette that was knocked into an adjacent intron for subsequent removal by cre-recombinase (Figure 1 and Supplementary Figure S2). This approach allowed us to obtain a homogenous population of fibroblasts by selection, as opposed to non-selection-based strategies that have observed gene correction rates of $<10 \%{ }^{45}$ This consideration is crucial given that the subsequent reprogramming process results in a sub-fraction of cells reprogrammed to pluripotency and, if the input is not uniform, extensive screening is mandated. Our target cell population was carefully considered due to the fact that fibroblasts competent for $\mathrm{C7}$ protein expression have utility for localised therapy in RDEB. ${ }^{49}$ Given the comparatively diminished proliferation capacity and rate of fibroblasts compared with iPSCs, we also attempted gene correction in iPSCs due to the fact that HDR occurs in late $S$ and G2. ${ }^{50,51}$ Surprisingly, we were unable to achieve gene correction in this patient's iPSCs, while we obtained 12 corrected fibroblast clones. One possible explanation for this is poor accessibility due to the chromatin status of iPSCs versus fibroblasts. Given that at the resolution of western blotting we were unable to show C7 protein expression in iPSCs (Supplementary Figure S7c), and the poor ability of nucleases to modify silent or repressed genes, ${ }^{52}$ we postulate that the 4317 position of the COL7A1 locus may be refractory to gene modification as a result of chromatin state.

The Sendai virus reprogramming method is a non-integrating RNA virus platform that mediates robust reprogramming frequencies without retaining the reprogramming factors by virtue of natural loss by dilution as the cells divide. ${ }^{53}$ As such, the need for secondary factors (e.g., cre-recombinase) to remove the viral footprint is eliminated, as is the risk for adverse events associated with the random integration of viral vectors. Using this methodology, we obtained numerous clones and chose two (11-2 and 11-5) for iPSC quality assurance and control assessment. Each clone exhibited morphology consistent with pluripotency (i.e., discrete colonies with rounded edges), were karyotypically normal (Supplementary Figure S3), and expressed pluripotencyassociated markers NANOG, TRA-60, -81 , SSEA-3, -4 and OCT-3/4 (Supplementary Figure S5). Transcriptional profiling and promoter methylation analysis further confirmed successful reprogramming, and iPSC clones implanted into immune-deficient animals gave rise to teratomas consisting of tissues representative of all three germ layers (Supplementary Figure S6). These data demonstrate the successful reprogramming of CRISPR/Cas9 gene-corrected fibroblasts to iPSCs, which were subsequently maintained and 
propagated in feeder-free conditions and served as a renewable template for the generation of keratinocytes, MSCs and HPSCs.

Using defined conditions, we were able to derive epidermal cells with a morphology consistent with that of keratinocytes, and these cells expressed the keratinocyte markers KRT5, KRT14, p63, COL17A1 and COL7A1 (Figure 2). These iPSC-derived keratinocytes hold promise for localised application to chronic and/or severe wounds-either alone or in support of systemic therapy.

MSCs have shown promise as both a localised and systemic therapeutic intervention in the setting of RDEB. However, genetic correction of MSCs has not been demonstrated, leading us to pursue in vitro production of MSCs from our gene-corrected iPSCs. By exposing iPSCs to bFGF, PDGF and EGF, and utilising ROCK inhibition during the early passages, we were able consistently produce robust MSC cultures (Supplementary Figure S7). These cells were morphologically identical to bone marrow-derived MSCs (Figure 3a and Supplementary Figure S7), and were able to be expanded and propagated over multiple passages while retaining surface expression of CD73, CD105 and CD90, and the ability to undergo chondro-, osteo- and adipo-genic differentiation in a manner identical to bone marrow-derived MSCs (Figures 3b-e and Supplementary Figure S7). Importantly, from $\sim 5 \times 10^{6}$ starting iPSCs, we were routinely able to generate $>1 \times 10^{8}$ iPSC-MSCs (data not shown). Considering that a recent clinical trial using systemic delivery of MSCs for treatment of RDEB reported a dose of $1-3 \times 10^{6} / \mathrm{kg}^{3}$, our protocol is capable of generating clinically relevant yields of iPSC-MSCs.

A highly challenging hurdle in stem cell biology remains the in vitro conversion of iPSCs to definitive HPSCs. Despite substantial effort, there remains no robust, efficient, or clinically translatable method for converting iPSCs into HPSCs capable of long-term engraftment. Towards this goal, we sought to synergise several of the most promising advancements in this area to achieve a more robust platform for producing definitive-type HPSCs. The first improvement involved optimising the basal media composition for robust production and growth of EBs from feeder-free iPSCs under fully defined conditions. This centred on the stepwise induction of mesoderm with BMP-4 and bFGF followed by specification to haematopoiesis with vascular endothelial growth factor, stem cell factor, IL-3 and Flt-3 ligand. Further steps were small molecule inhibition of the Activin/Nodal pathway with SB431542 with augmentation of the Wnt pathway ${ }^{36}$ via inhibition of GS3K $\beta$ with CHIR99021 to drive definitive haematopoiesis (Figures 4 and 5 and Supplementary Figure S9). Our hypothesis was that the culture conditions and modulation of the primitive and definitive fate determinant modulators with CHIR99021 and SB431542 would bias the cells away from primitive haematopoiesis and promote definitive haematopoietic commitment. However, even though EB-derived CD34+ hemogenic precursors were definitive in nature as evidenced by their capacity for T-lineage differentiation given the appropriate micro environmental cues, their intrinsic capacity for multilineage haematopoietic differentiation was limited in CFU assays. (Figure 4 and Supplementary Figure S9c). This prompted us to employ a vascular induction technique aimed at recapitulating the complicated embryonic haematopoietic niche environment ex vivo, to determine whether the instructive cues provided by the E4ORF1 transformed endothelial cells would promote maturation and expansion of EB-derived CD34+ cells to multilineage haematopoietic progenitors. In contrast to the data observed when EBs were utilised without vascular induction, the inclusion of the coculture system resulted in the substantial increase of both the progenitor frequency and multilineage capacity (Figures 4 and 5). These data outline a robust, reproducible strategy for the in vitro production of multilineage definitive HPSCs from iPSCs.

In conjunction with our corresponding data showing the ability to correct RDEB-causing mutations and employ an engineering strategy that maximises the potential of iPSCs for therapeutic cell generation these results hold great promise for RDEB and other maladies that may benefit from regenerative medicine.

\section{MATERIALS AND METHODS}

Research subject and cell line derivation

Patient-derived samples were obtained following parental consent and approval from the University of Minnesota Institutional Review Board. A $4 \mathrm{~mm}$ skin punch biopsy was collected followed by mincing the skin tissue and submerging in complete DMEM media with 20\% FBS, non-essential amino acids, antibiotics and glutamax all from Invitrogen, (Carlsbad, CA, USA). Primary fibroblasts were maintained in complete DMEM media under hypoxic $\left(<2 \% \mathrm{O}_{2}\right)$ conditions.

\section{Mice}

NOD/SCID IL2rycnull (NSG) mice were ordered from Jackson Laboratories (Bar Harbor, ME, USA). All animal studies were approved by the University of Minnesota Institutional Animal Care and Use Committee.

\section{CRISPR/Cas9 reagent and donor construction}

The S. pyogenes hCas9 plasmid was a gift from Dr George Church (Addgene plasmid \#41815), ${ }^{54}$ and the gRNAs were assembled into a plasmid with a U6 promoter and pollll termination signal. ${ }^{15}$ The donor sequence was comprised of left and right arms of homology that were assembled by amplification from the human genome with: Left arm F: $5^{\prime}$ CCTGACCTCTTCACCTCCTCAGGGCTTCC-3', Left Arm R: 5'-GGGCCACACCTC ACTCCCAAAGATACCAGG-3'. The Right arm was amplified with RT Arm1 F: 5'-AGGGTCATGGGGTCGTCATCTGTIITCTAGGG-3' and Reverse: 5'-AACTAT GAAGCCCAGCACCCAACCACTGCCCCAGG-3' that overlapped with a synthesised fragment containing the corrective base and two silent polymorphisms 5'-CTCTCCTGGGGCAGTGGTTGGGTGCTGGGCTTCATAGTTC TTGCTCATATITITACTCACTTCTTCCTAGGGTCTTCCTGGCAGCCCTGGACCCC AAGGCCCCGTTGGCCCCCCTGGAAAGAAAGGAGAAAAAGTAGGAAGGCTGAC TTGATGATGTCCCAGTTCTGGGGTGGGAGGCTGCGTGCTGGGGGCAGCCTCCC TTCGGTCTTCCCACCCGTGTGTITCTCCTTCAGGGTGACTC- 3 '. The remainder of the right donor arm was amplified with: Right Arm2 F: 5'-CACCCGTGTG TITCTCCTTCAGGGTGACTC-3' and reverse: $5^{\prime}$-GGGCAAGAAGTCAGAACCAG AAAGGGCACAGC-3'. These fragments were assembled into a plasmid containing the left donor arm followed by a floxed PGK puromycin cassette by Gibson assembly to complete the donor. ${ }^{55}$

\section{Gene transfer}

Primary fibroblasts $(200,000)$ were electroporated with $1 \mu \mathrm{g}$ each of the Cas 9 and gRNA plasmids and $5 \mu \mathrm{g}$ of the donor using the following settings on the Neon Transfection System (Invitrogen): 1500 V, 20 ms pulse width, and a single pulse. ${ }^{44}$

\section{Surveyor nuclease}

Genomic DNA was isolated $48 \mathrm{~h}$ after Cas9/gRNA electroporation and amplified for with Surveyor 13F (5'-CCATGACCCTCATCACTCCT-3') and Surveyor 708R primers ( $\left.5^{\prime}-\pi T T G G G G G T C A G A G A T T G-3^{\prime}\right)$ and incubated with the Surveyor nuclease (Integrated DNA Technologies, Coralville, IA, USA $)^{56}$ and resolved on a 10\% TBE PAGE gel (Invitrogen).

\section{Off-target analysis}

293T cells were transfected using Lipofectamine 2000 and the Cas9 nuclease or nickase $(500 \mathrm{ng})$ and guide RNA plasmid $(500 \mathrm{ng})$. Genomic DNA was isolated $72 \mathrm{~h}$ post gene transfer and PCR amplified with 4317 131F: 5'-TCCCAAAGTCCTTGAAATCC-3') and 4317 777R: 5'-GCCCACCATAT TCAGAATCC-3') for on-target site amplification. Off-target sites were identified using the MIT CRISPR Design Tool (http://crispr.mit.edu/) and were amplified with following primers: ACAP3F: $5^{\prime}$-ACGGCCTTGTACAG AACTGG-3', ACAP3R: 5'-GTGCTTTCGCTCCATCTCAC-3', GRK6F: 5'-CCAGAG GAGCCTTGAGTTGG-3', GRK6R: 5'-CTACCCAGCCCCCTTACTTC-3', E2F2F: 5'-TGGTACGTCGAGGGTCCTAA-3', E2F2R: 5'-CCTTGGAGGCTACTGACAGC-3', SEC23AF: 5'-GCTACCTCTCCTCCCTCCTC-3', and SEC23AR: 5'-CCACCGTTTC CACATCTIT-3', CARD10F: 5'-GGCTCATCCGTAACCTGCTA-3', CARD10R: 5'-GGGCAACCTGGAGATACAGA-3', SYTL1F: 5'-TाTTGTCGAGATGGGGTCTC -3', SYTL1R: 5'-GGGGACAGTGCATAATCTGG-3', FADS3F: 5'-AGATGAACCAC ATCCCCAAG-3', FADS3R: 5'-TGGACAAGGGTAGGCATAGG-3', FAM3DF: 5'-AA 
GAATCAGGAAGCCCAGGT-3', FAM3DR: 5'-GTCTCAAACAGCCCAGCTTC-3', MLLT1F: 5'-GAGACCAAGCTGGAAAGCAC-3', MLLT1R: 5'-AGCTCAGAACCTCA GGACCA-3', MYO1EF: 5'-CATTCCTCTCTGCCACCTTC-3', MYO1ER: 5'-TGTTC GCCGATTCCTITATT-3', TIE1F: 5'-AGAGGTGACACAGCCCTCAT-3', TIE1R: 5'-AGGGTCTTCTCCCAGTCAGG-3', SHANK2F: 5'-CTTTGGGTCCCTGTTGA GAC-3', SHANK2R: 5'-GAAGACGTGCTCCATCCCTA-3'. PCR conditions were: $94^{\circ} \mathrm{C} \times 2$ min followed by 40 cycles of $94^{\circ} \mathrm{C} \times 40 \mathrm{~s}, 58^{\circ} \mathrm{C} \times 40 \mathrm{~s}$, and $68^{\circ}$ $\mathrm{C} \times 1 \mathrm{~min}$ with AccuPrime DNA polymerase (ThermoFisher Scientific, Waltham, MA, USA). PCR products were denatured and renatured and assayed by Surveyor nuclease (Integrated DNA Technologies) and subsequent resolution on a 10\% TBE PAGE gel (ThermoFisher Scientific). ${ }^{156}$ All gel images used the same exposure times.

\section{Selection and HDR analysis}

$80 \%$ confluent fibroblasts that had undergone electroporation were exposed to $0.2 \mu \mathrm{g} / \mathrm{ml}$ puromycin and then plated at low density for clonal derivation. Individual cells were segregated in cloning disks and expanded for HDR analysis using an inside-out PCR. A primer inside the donor (5'-GCCACTCCCACTGTCCTITCCT-3') and outside the right homology arm (5'-GGGCAAGAAGTCAGAACCAG-3') were amplified, cloned into the pCR 4 TOPO vector (Invitrogen) and sequenced by the Sanger method to confirm HDR. CDNA correction was demonstrated by amplification of a product with CDNAF: 5'-GTGACAAAGGCGATCGTG-3' and CDNAR: 5'-GTCCCCGTG GGCCCTGC-3' followed by sequencing.

\section{PGK puromycin removal}

Cre-recombinase mRNA (TriLink Biotechnologies, San Diego, CA, USA) was electroporated into iPSC clones at a dose of $1 \mu \mathrm{g}$ using the conditions noted above, and excision was confirmed by PCR and Sanger sequencing.

\section{iPSC generation and teratoma assay}

Gene-corrected fibroblasts (or uncorrected cells as a control) were reprogrammed to iPSCs as described ${ }^{43,57}$ with Sendai virus delivery of the reprogramming factors. ${ }^{53}$ Karyotype, gene expression and immunofluorescence as part of quality assurance and control were also performed as detailed elsewhere. ${ }^{43,57}$ All iPSC lines were regularly tested for mycoplasma and all lines used in this study tested negative. iPSCs contained in matrigel were implanted onto the hind flank of NSG mice $(n=3-5)$ until a palpable mass formed. Teratoma tissue was excised for histological examination following embedding and staining by haematoxylin and eosin.

\section{Differentiation of iPSCs to keratinocytes}

iPSCs were maintained feeder-free on Geltrex-coated (ThermoFisher Scientific) tissue culture plastic in TesR1 (STEMCELL Technologies, Vancouver, BC, Canada). For keratinocyte differentiation, mid-passage iPSCs at $~ 50 \%$ confluency in six-well plates had media changed to defined keratinocyteSFM media supplemented with $25 \mathrm{ng} / \mathrm{ml}$ BMP-4 (Bio-Techne, Minneapolis, MN, USA) and $1 \mu$ M RA (STEMCELL Technologies) for the first $96 \mathrm{~h}$, at which point the BMP4 and RA were removed, followed by media changes every $72 \mathrm{~h}$ thereafter until epithelial cell morphology became apparent ( 10 days). At this timepoint, the media was switched to Cnt-07 (CELLnTEC, Bern, Switzerland) and the cells cultured until confluency. At this point they were pre-treated with ROCK inhibitor (Y-27632, VWR) for at least $1 \mathrm{~h}$ and passaged using Accutase (STEMCELL Technologies) onto $10 \mathrm{~cm}^{2}$ tissue culture plates coated with collagen I/collagen IV, where rapid attachmentmediated enrichment of Krt14+ cells was performed as previously described. Resultant iPSC-keratinocyte cultures were cultured in Cnt-07 media containing $10 \mu \mathrm{M}$ ROCK inhibitor until first media change after plating (CELLnTEC).

\section{Differentiation of iPSCs to MSCs}

iPSCs were maintained feeder-free on Geltrex-coated tissue culture plastic in TesR1 (STEMCELL Technologies). Differentiation to MSCs was initiated by transferring mid-passage iPSCs at 50\% confluency to MSC medium, which consisted of Minimal Essential Medium Alpha supplemented with $5 \%$ fetal bovine serum, $5 \%$ horse serum and $10 \mathrm{ng} / \mathrm{ml}$ each of human PDGF-AB, EGF, and bFGF (all from PeproTech, Rocky Hill, NJ, USA). Media was changed every $48 \mathrm{~h}$ until cells with fibroblastic morphology were apparent and cultures neared confluency. At this point, MSC cultures were pretreated with $10 \mu \mathrm{M}$ ROCK inhibitor for at least $1 \mathrm{~h}$ and dissociated using a
50:50 mixture of Accutase and 0.25\% Trypsin-EDTA incubated at $37^{\circ} \mathrm{C}$. When cells had detached, the Accutase/trypsin mixture was diluted with $2 \times$ volume of PBS $+1 \%$ FBS +3 mm EDTA to prevent clumping. Cells were centrifuged at $400 \mathrm{~g}$ for $5 \mathrm{~min}$ and re-plated onto gelatin-coated plates in growth media plus cytokines containing $5 \mu \mathrm{M}$ ROCK inhibitor to enhance plating efficiency. Between passages 3-5, cultures can be weaned off of ROCK inhibitor during passage. Flow cytometry analysis was performed using the following antibodies, all from eBioscience (San Diego, CA, USA): Anti-Human CD73 eFluor 450, 48-0739; anti-Human CD105 (Endoglin) PE, 12-1057; and anti-Human CD90 (Thy-1) APC, 17-0909.

\section{Maintenance and differentiation of human iPSCs to} haematopoietic progenitors

Human iPSC lines were maintained on Matrigel- or Geltrex-coated plastic ware in TesR1 (STEMCELL Technologies). For differentiation, hiPSCs were cultured at $~ 80-90 \%$ confluency, followed by EB generation, as described previously. ${ }^{37,38}$ Briefly, the undifferentiated hiPSCs were dissociated with Accutase (STEMCELL Technologies) treatment. Aggregates were resuspended in APEL-differentiation medium (STEMCELL Technologies), supplemented with BMP-4 $(20 \mathrm{ng} / \mathrm{ml})$ and bFGF $(5 \mathrm{ng} / \mathrm{ml})$ and cultured in nontissue culture treated plates. After $42 \mathrm{~h}$, developing EBs were collected and resuspended in APEL-differentiation media supplemented with BMP-4, bFGF, CHIR99021 (3 $\mu \mathrm{M}$, Stemgent, Lexington, MA, USA), and SB431542 ( $6 \mu \mathrm{M}$, Selleck Chemicals, Houston, TX, USA).

After $24 \mathrm{~h}$, EBs were collected and resuspended in either $60 \%$ APEL-differentiation medium $+40 \%$ STEMSPAN II medium (STEMCELL Technologies) for vascular induction, or $100 \%$ APEL-differentiation medium for T-lineage differentiation; both supplemented with VEGF $(20 \mathrm{ng} / \mathrm{ml})$, bFGF $(5 \mathrm{ng} / \mathrm{ml}), \mathrm{IL}-3(20 \mathrm{ng} / \mathrm{ml})$, Flt $3 \mathrm{~L}(20 \mathrm{ng} / \mathrm{ml})$ and SCF $(100 \mathrm{ng} / \mathrm{ml})$ and cultured for another 5-6 days. Cultures were maintained in a $5 \% \mathrm{CO}_{2} / 5 \%$ $\mathrm{O}_{2} / 90 \% \mathrm{~N}_{2}$ environment. At day 8 or 9 , EBs were harvested, washed once with PBS, and dissociated using Accutase and $0.25 \%$ trypsin EDTA mixture until no visible clumps observed. CD34+ cells were enriched using EasySep CD34+ isolation kit (STEMCELL Technologies). Flow cytometry analysis was performed using the following antibodies, all from eBioscience: AntiHuman CD45 APC-eFluor 780, 47-0459; anti-Human CD34 APC, 17-0349-42; anti-Human CD43 PE, 12-0439; anti-Human CD73 eFluor 450, 48-0739; antiHuman CD184 (CXCR4) PE-Cy7, 25-9999-42.

\section{Vascular induction}

For vascular induction, $1 \times 10^{5}$ purified EB-derived CD34+ cells were plated onto $85 \%$ confluent VeraVec HUVEC (Angiocrine Bioscience, San Diego, CA, USA) in StemSpan II supplemented with the following: SCF $(200 \mathrm{ng} / \mathrm{ml})$, Flt3L $(10 \mathrm{ng} / \mathrm{ml})$, TPO $(30 \mathrm{ng} / \mathrm{ml}), \mathrm{IL}-11(5 \mathrm{ng} / \mathrm{ml}), \mathrm{IGF}-1(25 \mathrm{ng} / \mathrm{ml}), \mathrm{bFGF}$ $(5 \mathrm{ng} / \mathrm{ml})$, VEGF $(5 \mathrm{ng} / \mathrm{ml})$, EPO $(2 \mathrm{lU} / \mathrm{ml}), \mathrm{IL}-6(10 \mathrm{ng} / \mathrm{ml}), \mathrm{IL}-3(30 \mathrm{ng} / \mathrm{ml})$, BMP-4 $(10 \mathrm{ng} / \mathrm{ml})$ and losartan $(100 \mu \mathrm{M})$. Co-cultures were maintained for 7-9 days.

\section{T-lineage differentiation}

For $\mathrm{T}$ lineage differentiation, $1 \times 10^{5}$ purified CD34+ cells were plated onto confluent OP9-DLL4 cells for about 3-4 weeks and passaged every $4-5$ days as described previously. ${ }^{35}$ All recombinant factors are human and were purchased from R\&D Systems (Minneapolis, MN, USA).

\section{Colony-forming unit assay}

Cells were placed in MethoCult according to the manufacturer's instructions (STEMCELL Technologies). Colonies were enumerated by an experienced analyst using light microscopy at low magnification ( $\times 4$ objective).

\section{Western blotting}

Cellular lysates in RIPA lysis buffer (ThermoFisher Scientific) were electrophoresed using the XCell SureLock Mini-Cell Electrophoresis System with a NuPAGE Novex 3-8\% acetate gel (ThermoFisher Scientific). Proteins were transferred to a nitrocellulose membrane and stained with the 4D2 mouse monoclonal anti-human collagen 7 antibody (Santa Cruz Biotechnology, Dallas, TX, USA). Secondary staining was performed with a goat anti-mouse horseradish peroxidase conjugated antibody (Santa Cruz Biotechnology) and detection with the SuperSignal West Pico Chemiluminescent Substrate (ThermoFisher Scientific). 


\section{Statistics}

Statistical differences in survival were determined by Log-rank test using Prism software (GraphPad Software, La Jolla, CA, USA). All other statistical analyses were performed using the Student's t-test with $P$ values less than 0.5 being considered significant. No statistical methods were used to predetermine the sample sizes. The experiments were not randomized and the investigators were not blinded.

\section{ACKNOWLEDGEMENTS}

We are grateful to the patients, their families, and the patient support organizations that made this work possible. We are grateful to Nancy Griggs Morgan for invaluable assistance in manuscript preparation and editing. BRW is supported by $\mathrm{NIH}$ T32- HL007062. We are also thankful for the generosity of the Lindahl family, the Children's Cancer Research Fund and the Corrigan Family. MJO is supported by 8UL1TR000114-02. JT is supported in part by R01 AR063070 and P01 CA065493. The present work was partly supported by research funds from the National Research Foundation of Korea (NRF-2015K1A4A3046807). Research reported in this publication was supported by the National Center for Advancing Translational Sciences of the National Institutes of Health Award Number UL1TR000114 (MJO). The content is solely the responsibility of the authors and does not necessarily represent the official views of the National Institutes of Health.

\section{CONTRIBUTIONS}

BRW, MJO, ANM, KT, C-IL, APD, LX, CE, CJL, RTM, MJR and DDP designed and performed the experiments, analysed the data and wrote the paper. DDP and BRW are the major contributors who, along with MJO conceived, designed and established the protocol to generate CD34+ haematopoietic cells. DDP played the major role, along with BRW, to establish T-cell generation methods. CJK, BRB and JT supervised the research, designed the experiments and wrote the manuscript.

\section{COMPETING INTERESTS}

The authors declare no conflict of interest.

\section{REFERENCES}

1. van den Akker, P. C. et al. The International Dystrophic Epidermolysis Bullosa Patient Registry: an online database of dystrophic epidermolysis bullosa patients and their COL7A1 mutations. Hum. Mutat. 32, 1100-1107 (2011).

2. Wagner, J. E. et al. Bone marrow transplantation for recessive dystrophic epidermolysis bullosa. N. Engl. J. Med. 363, 629-639.

3. Petrof, G. et al. Potential of systemic allogeneic mesenchymal stromal cell therapy for children with recessive dystrophic epidermolysis bullosa. J Invest Dermatol 135, 2319-2321 (2015).

4. Latifi-Pupovci, H. et al. In vitro migration and proliferation ('wound healing') potential of mesenchymal stromal cells generated from human CD271(+) bone marrow mononuclear cells. J. Transl. Med. 13, 315 (2015).

5. Wagner, J. E. et al. Bone marrow transplantation for recessive dystrophic epidermolysis bullosa. N. Engl. J. Med. 363, 629-639 (2010).

6. Petrof, G., Martinez-Queipo, M., Mellerio, J. E., Kemp, P. \& McGrath, J. A. Fibroblast cell therapy enhances initial healing in recessive dystrophic epidermolysis bullosa wounds: results of a randomized, vehicle-controlled trial. Br. J. Dermatol. 169, 1025-1033 (2013).

7. Woodley, D. T. et al. Intradermal injection of lentiviral vectors corrects regenerated human dystrophic epidermolysis bullosa skin tissue in vivo. Mol. Ther. 10, 318-326 (2004).

8. Georgiadis, C. et al. Lentiviral engineered fibroblasts expressing codon-optimized COL7A1 restore anchoring fibrils in RDEB. J. Invest. Dermatol. 136, 284-292 (2016).

9. Hacein-Bey-Abina, S. et al. Insertional oncogenesis in 4 patients after retrovirusmediated gene therapy of SCID-X1. J. Clin. Invest. 118, 3132-3142 (2008).

10. Christian, M. et al. Targeting DNA double-strand breaks with TAL effector nucleases. Genetics 186, 757-761 (2010).

11. Porteus, M. H. \& Carroll, D. Gene targeting using zinc finger nucleases. Nat. Biotechnol. 23, 967-973 (2005).

12. Paques, F. \& Duchateau, P. Meganucleases and DNA double-strand break-induced recombination: perspectives for gene therapy. Curr. Gene Ther. 7, 49-66 (2007).

13. Cong, L. et al. Multiplex genome engineering using CRISPR/Cas systems. Science 339, 819-823 (2013).

14. Shen, B. et al. Efficient genome modification by CRISPR-Cas9 nickase with minimal off-target effects. Nat. Methods 11, 399-402 (2014).
15. Osborn, M. J. et al. Fanconi anemia gene editing by the CRISPR/Cas9 system. Hum. Gene Ther. 26, 114-126 (2015).

16. Bannwarth, S., Procaccio, V. \& Paquis-Flucklinger, V. Surveyor nuclease: a new strategy for a rapid identification of heteroplasmic mitochondrial DNA mutations in patients with respiratory chain defects. Hum. Mutat. 25, 575-582 (2005).

17. Stelzl, U. et al. A human protein-protein interaction network: a resource for annotating the proteome. Cell 122, 957-968 (2005).

18. Itoh, M., Kiuru, M., Cairo, M. S. \& Christiano, A. M. Generation of keratinocytes from normal and recessive dystrophic epidermolysis bullosa-induced pluripotent stem cells. Proc. Natl Acad. Sci. USA 108, 8797-8802 (2011).

19. Umegaki-Arao, N. et al. Induced pluripotent stem cells from human revertant keratinocytes for the treatment of epidermolysis bullosa. Sci. Transl. Med. 6, 264ra164 (2014).

20. Kogut, I., Roop, D. R. \& Bilousova, G. Differentiation of human induced pluripotent stem cells into a keratinocyte lineage. Methods Mol. Biol. 1195, 1-12 (2014).

21. Conget, P. et al. Replenishment of type VII collagen and re-epithelialization of chronically ulcerated skin after intradermal administration of allogeneic mesenchymal stromal cells in two patients with recessive dystrophic epidermolysis bullosa. Cytotherapy 12, 429-431 (2010).

22. Sasaki, M. et al. Mesenchymal stem cells are recruited into wounded skin and contribute to wound repair by transdifferentiation into multiple skin cell type. J. Immunol. 180, 2581-2587 (2008).

23. Schwarz, S. et al. Bone marrow-derived mesenchymal stem cells migrate to healthy and damaged salivary glands following stem cell infusion. Int. J. Oral Sci. 6, 154-161 (2014).

24. Wu, Y., Chen, L., Scott, P. G. \& Tredget, E. E. Mesenchymal stem cells enhance wound healing through differentiation and angiogenesis. Stem Cells 25, 2648-2659 (2007).

25. Fu, X. et al. Comparison of immunological characteristics of mesenchymal stem cells derived from human embryonic stem cells and bone marrow. Tissue Eng. Part A 21, 616-626 (2015).

26. Jacobs, S. A., Roobrouck, V. D., Verfaillie, C. M. \& Van Gool, S. W. Immunological characteristics of human mesenchymal stem cells and multipotent adult progenitor cells. Immunol. Cell Biol. 91, 32-39 (2013).

27. Lian, Q. et al. Functional mesenchymal stem cells derived from human induced pluripotent stem cells attenuate limb ischemia in mice. Circulation 121, 1113-1123 (2010)

28. Dominici, M. et al. Minimal criteria for defining multipotent mesenchymal stromal cells. The International Society for Cellular Therapy position statement. Cytotherapy 8, 315-317 (2006).

29. Tolar, J. et al. Amelioration of epidermolysis bullosa by transfer of wild-type bone marrow cells. Blood 113, 1167-1174 (2009).

30. Fujita, Y. et al. Bone marrow transplantation restores epidermal basement membrane protein expression and rescues epidermolysis bullosa model mice. Proc. Natl Acad. Sci. USA 107, 14345-14350 (2010).

31. Genovese, P. et al. Targeted genome editing in human repopulating haematopoietic stem cells. Nature 510, 235-240 (2014).

32. Hoban, M. D. et al. Correction of the sickle cell disease mutation in human hematopoietic stem/progenitor cells. Blood 125, 2597-2604 (2015).

33. Wang, J. et al. Homology-driven genome editing in hematopoietic stem and progenitor cells using ZFN mRNA and AAV6 donors. Nat. Biotechnol. 33, 1256-1263 (2015).

34. Ditadi, A. et al. Human definitive haemogenic endothelium and arterial vascular endothelium represent distinct lineages. Nat. Cell Biol. 17, 580-591 (2015).

35. Kennedy, M. et al. T lymphocyte potential marks the emergence of definitive hematopoietic progenitors in human pluripotent stem cell differentiation cultures. Cell Rep. 2, 1722-1735 (2012).

36. Sturgeon, C. M., Ditadi, A., Awong, G., Kennedy, M. \& Keller, G. Wnt signaling controls the specification of definitive and primitive hematopoiesis from human pluripotent stem cells. Nat. Biotechnol. 32, 554-561 (2014).

37. Ng, E. S., Davis, R., Stanley, E. G. \& Elefanty, A. G. A protocol describing the use of a recombinant protein-based, animal product-free medium (APEL) for human embryonic stem cell differentiation as spin embryoid bodies. Nat. Protoc. 3, 768-776 (2008).

38. Osborn, M. et al. CRISPR/Cas9 Targeted Gene Editing and Cellular Engineering in Fanconi Anemia. Stem Cells Dev. 25, 1591-1603 (2016).

39. Schmitt, T. M. et al. Induction of T cell development and establishment of T cell competence from embryonic stem cells differentiated in vitro. Nat. Immunol. 5, 410-417 (2004).

40. Gori, J. L. et al. Vascular niche promotes hematopoietic multipotent progenitor formation from pluripotent stem cells. J. Clin. Invest. 125, 1243-1254 (2015).

41. Sandler, V. M. et al. Reprogramming human endothelial cells to haematopoietic cells requires vascular induction. Nature 511, 312-318 (2014). 
42. Kuhl, T. et al. High local concentrations of intradermal MSCs restore skin integrity and facilitate wound healing in dystrophic epidermolysis bullosa. Mol. Ther. 23, 1368-1379 (2015).

43. Tolar, J. et al. Induced pluripotent stem cells from individuals with recessive dystrophic epidermolysis bullosa. J. Invest. Dermatol. 131, 848-856 (2011).

44. Osborn, M. J. et al. TALEN-based gene correction for epidermolysis bullosa. Mol. Ther. 21, 1151-1159 (2013).

45. Izmiryan, A., Danos, O. \& Hovnanian, A. Meganuclease-mediated COL7A1 gene correction for recessive dystrophic epidermolysis bullosa. J. Invest. Dermatol. 136, 872-875 (2016).

46. Sebastiano, V. et al. Human COL7A1-corrected induced pluripotent stem cells for the treatment of recessive dystrophic epidermolysis bullosa. Sci. Transl. Med. 6, 264ra163 (2014).

47. Chamorro, C. et al. Gene editing for the efficient correction of a recurrent COL7A1 mutation in recessive dystrophic epidermolysis bullosa keratinocytes. Mol. Ther. Nucleic Acids 5, e307 (2016).

48. Wenzel, D. et al. Genetically corrected iPSCs as cell therapy for recessive dystrophic epidermolysis bullosa. Sci. Transl. Med. 6, 264ra165 (2014).

49. Goto, M. et al. Fibroblasts show more potential as target cells than keratinocytes in COL7A1 gene therapy of dystrophic epidermolysis bullosa. J. Invest. Dermatol. 126, 766-772 (2006).

50. Lin, S., Staahl, B. T., Alla, R. K. \& Doudna, J. A. Enhanced homology-directed human genome engineering by controlled timing of CRISPR/Cas9 delivery. Elife 3, e04766 (2014).

51. Heyer, W. D., Ehmsen, K. T. \& Liu, J. Regulation of homologous recombination in eukaryotes. Annu. Rev. Genet. 44, 113-139 (2010).
52. van Rensburg, R. et al. Chromatin structure of two genomic sites for targeted transgene integration in induced pluripotent stem cells and hematopoietic stem cells. Gene Ther. 20, 201-214 (2013).

53. Fujie, Y. et al. New type of sendai virus vector provides transgenefree iPS cells derived from chimpanzee blood. PLOS ONE 9, e113052 (2014).

54. Mali, P. et al. RNA-guided human genome engineering via Cas9. Science 339, 823-826 (2013).

55. Gibson, D. G. et al. Enzymatic assembly of DNA molecules up to several hundred kilobases. Nat. Methods 6, 343-345 (2009).

56. Guschin, D. Y. et al. A rapid and general assay for monitoring endogenous gene modification. Methods Mol. Biol. 649, 247-256 (2010).

57. Tolar, J. et al. Keratinocytes from induced pluripotent stem cells in junctional epidermolysis bullosa. J. Invest. Dermatol. 133, 562-565 (2013).

(i) This work is licensed under a Creative Commons Attribution 4.0 International License. The images or other third party material in this article are included in the article's Creative Commons license, unless indicated otherwise in the credit line; if the material is not included under the Creative Commons license, users will need to obtain permission from the license holder to reproduce the material. To view a copy of this license, visit http://creativecommons.org/licenses/ by $/ 4.0 /$

(C) The Author(s) 2016

Supplementary Information accompanies the paper on the npj Regenerative Medicine website (http://www.nature.com/npjregenmed) 